\title{
Numerical Investigations of the Effects of Sidewall Compression and Relaminarization in 3D Scramjet Inlet
}

\author{
Tue Nguyen*, Marek Behr ${ }^{\dagger}$ and Birgit U. Reinartz ${ }^{\ddagger}$ \\ Chair for Computational Analysis of Technical Systems, Center for Computational Engineering Science \\ RWTH Aachen University, 52056 Aachen, Germany \\ Oliver Hohn ${ }^{\S}$ and Ali Gülhan ${ }^{ף}$ \\ Institute of Aerodynamics and Flow Technology, Supersonic and Hypersonic Technology Department \\ German Aerospace Centre (DLR), Linder Höhe, 51147 Cologne, Germany
}

\begin{abstract}
This paper presents the numerical simulations and performance analysis of a 3D scramjet inlet with focus on the effects of sidewall compression and relaminarization. A wellvalidated finite volume flow solver was used to simulate a scramjet inlet with a double ramp configuration for outer compression and varying degrees of sidewall compression. The computed wall pressure and heat transfer in the symmetry plane are in close agreement with the measurements and numerical results indicate that sidewall compression alters the inlet performance significantly. The effects of relaminarization over the expansion corner prior to the interior part of the inlet is isolated and studied in both experiment and simulation.
\end{abstract}

\section{Introduction}

This work is a part of an ongoing research on supersonic combustion ramjet (scramjet) engines in Germany. ${ }^{1}$ A scramjet inlet consists of several external compression ramps and an interior part. The flow is compressed through a series of oblique shock waves generated by the ramps and the sidewalls. The large adverse pressure gradient produced by the shock waves may cause the boundary layer to separate and result in a considerable increase in pressure losses and blockage of flow into the interior part. It is important in the early design phase to be able to quantify the performance of an inlet in terms of compression ratio, mass flow capture and the flow conditions at the interface with the combustion chamber. Numerical computation offers a practical and efficient means by which this requirement can be met.

This paper focuses on two main topics. The first topic is a numerical study on the effects of sidewall compression (SWC) in a scramjet inlet. Designing an inlet with sidewall compression is a compromise between a longer inlet with smaller compression angle, thus imposing a penalty on size and weight to obtain the required compression and a shorter inlet with larger compression angle, which lead to stronger shocks from the sidewall and the increased probability of boundary layer separation. Holland ${ }^{2}$ showed that, when the location of the cowl is fixed, increasing the sidewall compression enhanced the total pressure recovery and the kinetic energy efficiency. However, the mass flow captured was reduced significantly. In this work, sidewall compression was achieved by introducing a smooth contraction along the outer part of the inlet.

*Ph.D. Student, Chair for Computational Analysis of Technical Systems, RWTH Aachen University, email: nguyen@cats.rwth-aachen.de, Member AIAA.

$\dagger$ Professor, Chair for Computational Analysis of Technical Systems, RWTH Aachen University.

${ }^{\ddagger}$ Research Fellow, Chair for Computational Analysis of Technical Systems, RWTH Aachen Univeristy, Senior Member AIAA.

$\S$ Ph.D. Student, Institute of Aerodynamics and Flow Technology, Supersonic and Hypersonic Technology Department, German Aerospace Centre (DLR), Member AIAA.

I Head of Department, Institute of Aerodynamics and Flow Technology, Supersonic and Hypersonic Technology Department, German Aerospace Centre (DLR), Member AIAA. 
The numerical results are first validated by comparing against the experimental data, then are used to gain some understanding on the effects of sidewall compression.

The second topic is a combined experimental and numerical study which aims to assess the ability of common turbulence models to predict rapid expansion in hypersonic flows. This phenomenon normally takes place at the entrance of the interior part where the flow is being expanded along a convex corner or surface. Since turbulence is damped by the flow expansion and acceleration and become less important to the development of the mean flow, the flow is normally considered as being "relaminarizing" (though turbulence does not completely vanish). The initially turbulent boundary layer returns to a laminar-like state in terms of velocity profile, skin friction and heat transfer. In supersonic flows, relaminarization was observed in the accelerating flow around an expansion corner ${ }^{3},{ }^{4}$ Recent numerical simulations ${ }^{5}$ have shown that common turbulence models can replicate the mean flow features and the Reynolds stresses reasonably accurately for Mach number up to 3. In this paper, the validity of this statement in the hypersonic regime will be verified.

\section{Experiment}

The scramjet inlet used for simulation in this work was designed at the German Aerospace Center (DLR) in Cologne as a part of the Research Training Group GRK 1095 "Aero-Thermodynamic Design of a Scramjet Propulsion System for Future Space Transportation Systems" in Germany. ${ }^{6}$ Schematic drawing of the inlet is shown in Fig. 1a. The inlet is $585 \mathrm{~mm}$ long and $100 \mathrm{~mm}$ wide and comprises of two external compression ramps and an interior part. The first ramp and the second ramp are inclined to the horizontal surface by $9^{\circ}$ and $20.5^{\circ}$, respectively. The leading edges of the first ramp and the cowl lip are blunt. The second ramp and the interior part is connected by an isentropic expansion surface. Sidewall compression was achieved by mounting a 3D insert ${ }^{7}$ onto the original sidewall as illustrated in Fig. 1b. This 3D insert provides a smooth contraction in the outer part of the inlet and results in a reduced width of the interior part. Two different 3D inserts were used: an 80-mm 3D insert and a 70-mm 3D insert that reduce the width to $80 \mathrm{~mm}$ and 70 $\mathrm{mm}$, respectively. These reductions are equivalent to sweep angles of approximately $1.7^{\circ}$ and $2.5^{\circ}$ along the outer part of the inlet.

In the relaminarization study, 3D insert was not used and the width remains constant at $100 \mathrm{~mm}$ from the leading edge of the inlet to the back of the interior part. Furthermore, the cowl was removed in order to isolate the effects of relaminarization. Heat transfer upstream and downstream of the curved expansion corner was deduced from infrared thermography and the results are used to validate the computations. The dimensionless Stanton number is defined as follows:

$$
S t=\frac{\dot{q}_{\operatorname{conv}}}{\rho_{\infty} V_{\infty} C_{p, a i r}\left(T_{r e c}-T_{w}\right)}
$$

in which $\dot{q}_{\text {conv }}$ is the convective heat flux, $\rho_{\infty}$ and $V_{\infty}$ are the free-stream density and velocity, $C_{p, a i r}$ is the specific heat capacity of air at constant pressure and $T_{r e c}$ and $T_{w}$ are the recovery and wall temperatures. The recovery temperature is given as

$$
T_{\text {rec }}=\left(1+r \frac{\kappa-1}{2} M_{\infty}^{2}\right) T_{\infty}
$$

where $\kappa$ is the heat capacity ratio and $M_{\infty}$ and $T_{\infty}$ are the free-stream Mach number and temperature. $r=0.9$ is the recovery factor.

The inlet was tested in the Hypersonic Wind Tunnel H2K at the DLR in Cologne. This facility is a blow down wind tunnel designed to simulate different Mach numbers and Reynolds numbers using contoured nozzles. Two inflow conditions were used in the experiments and are listed in Table 1. Condition A was used for both studies of sidewall compression and relaminarization. Condition B was only used for the study of relaminarization in order the assess the effect of Reynolds number on this phenomenon. In the experiments, pressure was measured by static pressure ports and Stanton number was deduced based on the data from the infrared thermography pictures. The uncertainties of the pressure coefficients and the Stanton number are $\pm 3.8-5.6 \%$ and $\pm 20 \%$, respectively. ${ }^{8}$ The exact dimensions of the inlet, the performance of the wind tunnel and the detailed measurement techniques can be found in Hohn and Gülhan. ${ }^{8}$ 


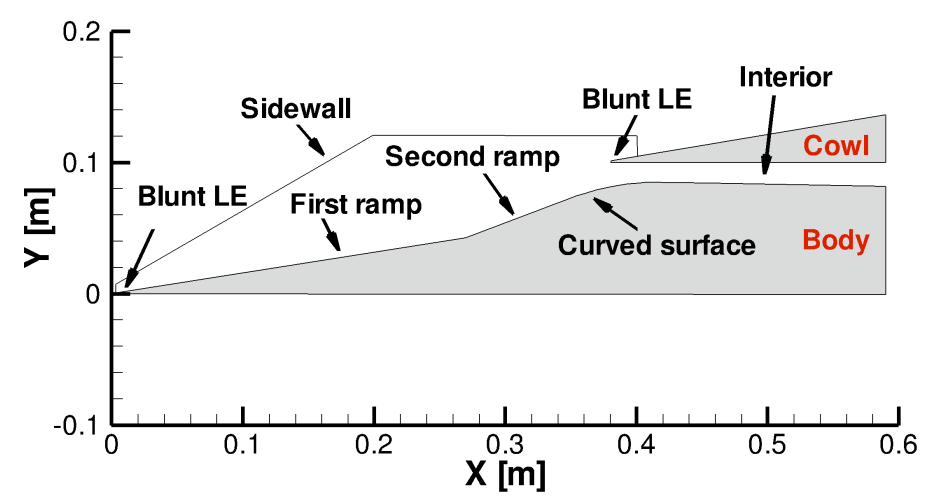

(a)

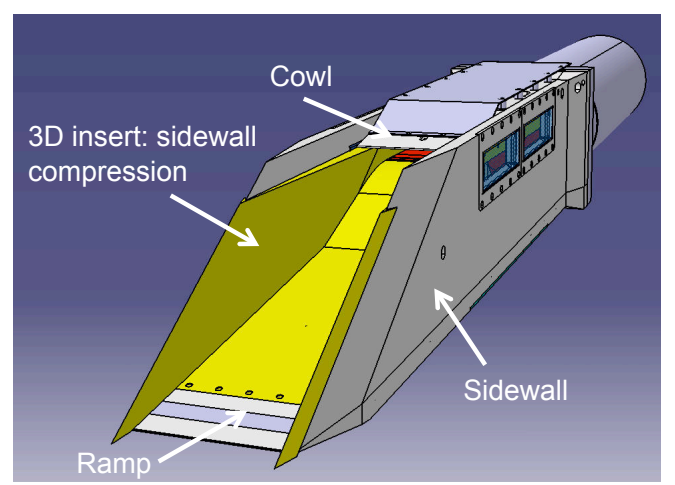

(b)

Figure 1: (a): Schematic drawing of symmetry plane of the inlet. (b): Inlet with mounted 3D insert.

\begin{tabular}{|l|l|l|l|l|l|}
\hline Cond. & $\mathrm{M}_{\infty}[-]$ & $\mathrm{Re}_{\infty, m}[1 / \mathrm{m}]$ & $\mathrm{P}_{0}[\mathrm{bar}]$ & $\mathrm{T}_{0}[\mathrm{~K}]$ & $\mathrm{T}_{w}[\mathrm{~K}]$ \\
\hline $\mathrm{A}$ & 7.0 & $4 \times 10^{6}$ & 7 & 500 & 300 \\
\hline $\mathrm{B}$ & 7.0 & $10 \times 10^{6}$ & 28.5 & 630 & 300 \\
\hline
\end{tabular}

Table 1: Inflow and boundary conditions in the experiments.

\section{Numerical Method}

The computations were performed using QUADFLOW code, which solves the RANS equations in 2D and 3D. The spatial discretization of the governing equations is based on a cell-centered finite volume scheme suited for unstructured grids. In this study, an AUSMDV upwind method ${ }^{9}$ is used to discretize the convective fluxes and second-order accuracy is achieved by a linear reconstruction. The viscous fluxes are discretized by quasi-central differencing. The mean flow equations are integrated in time by an explicit five-stage RungeKutta scheme. QUADFLOW has been validated extensively with different test cases consisting of various flow conditions. ${ }^{10}$ The flow solver has been proved to be suitable for supersonic/hypersonic computations. ${ }^{11}$

Turbulence closure is achieved with the 2-equation Shear Stress Transport (SST) k- $\omega$ model. ${ }^{12}$ Laminarturbulent transition was found experimentally around the separation bubble at the kink between the first ramp and the second ramp. ${ }^{13}$ This phenomenon is modeled by setting the production of turbulence kinetic energy in all cells on the first ramp to zero, thus, the flow is effectively laminar in this area. It will be shown later that this approach, which is named "transition box" here, provides reasonable agreement with respect to experimental data.

The following boundary conditions are used in the computations: supersonic inflow, supersonic outflow and solid wall. At supersonic inflow boundaries, the values are prescribed using the experimental data. The free-stream turbulence intensity was fixed at $0.5 \%$. At supersonic outflow boundaries, the variables are extrapolated from the interior assuming zero-gradient. At solid walls, the no-slip condition is enforced and isothermal condition is used. The turbulence kinetic energy is set to zero at the wall and the respective length scale is prescribed based on the first grid spacing according to Menter. ${ }^{12}$

\section{Grid Generation}

The grid used in this work was originally created by Krause and Ballmann ${ }^{14}$ for the inlet without sidewall compression using a multi-block elliptic structured grid generator $\left(\right.$ MegaCads $\left.^{15}\right)$. The grid was further refined and modified for the geometries with sidewall compression in this work. The final grid represents half of the inlet and consists of approximately 3 million cells. It should be noted that, for the computations with sidewall compression, only part of the intake is simulated. This part is from the leading edge of the ramp to the end of the 3D insert $(\mathrm{x}=440 \mathrm{~mm})$. 
The grid points in the wall normal direction are stretched by Poisson distribution in order to achieve $\mathrm{y}^{+}$smaller than 1 close to the wall everywhere in the computational domain (the minimum distance to the wall is $10^{-6}$ meter). The transverse grid lines were created in such a way that they are almost always perpendicular to the wall surface (Fig. 2). At blunt leading edge, great care was taken to make sure that the grid lines approximately align with the bowl shock and the grid resolution is good enough to resolve the shock wave, see Fig. 3a. Due to geometric complexity, the leading edge of the cowl lip is assumed to be sharp instead of blunt and the grid lines were clustered in this area to capture the lip shock (Fig. 3b).

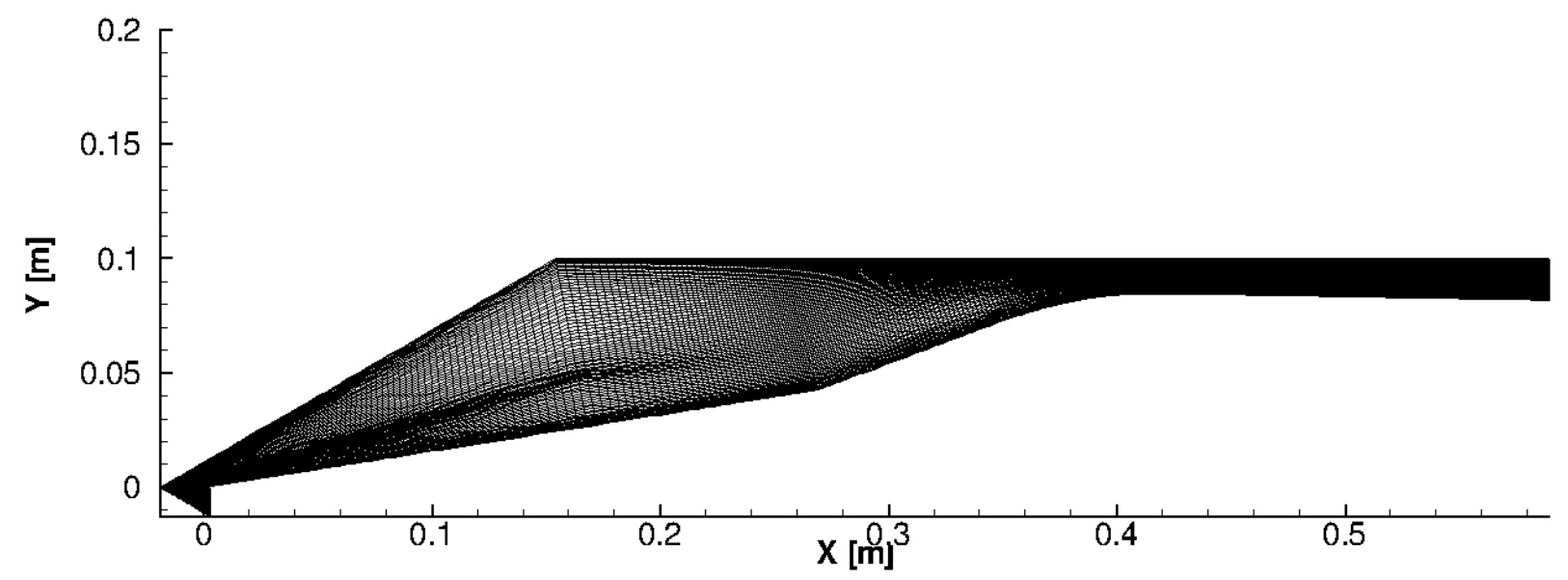

Figure 2: Grid distribution in the symmetry plane of the inlet.

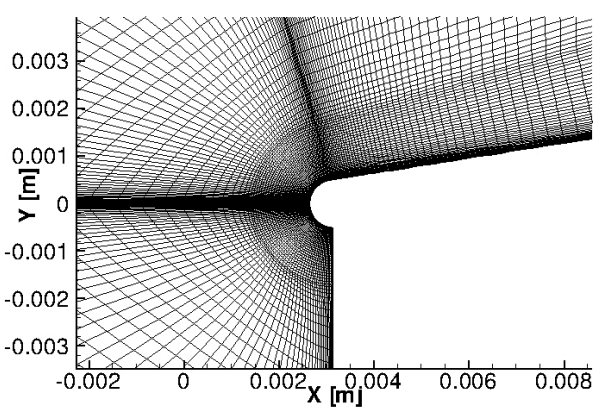

(a)

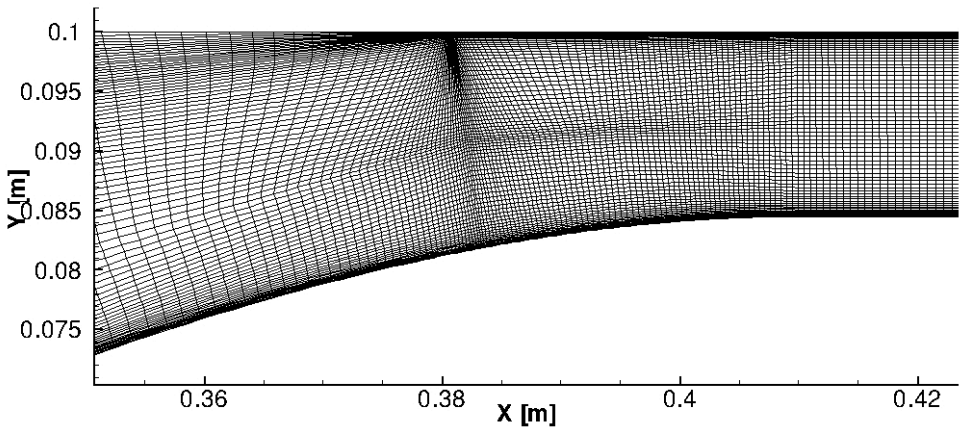

(b)

Figure 3: (a): Grid distribution around the blunt leading edge of the first ramp (b): Grid distribution at the entrance of the interior part.

Fig. 4 shows the 3D grids of the inlet with and without sidewall compression. It can be seen that the grid lines in the spanwise direction are also stretched towards the sidewall in order to resolve the boundary layer there.

\section{Baseline Configuration and General Flow Features}

In this section, the flow inside the inlet without 3D insert will be examined. Within this context, this configuration is referred to as the baseline configuration which has been studied experimentally and numerically in the past ${ }^{6,16,}{ }^{14}$ The general flow features in the symmetry plane of this configuration are illustrated in the bottom plot in Fig. 5. The flow is first compressed by the bow shock from the blunt leading edge of the first ramp. The separation bubble in between the first and second ramps is associated with a separation shock wave and a reattachment shock wave. At the entrance of the interior part, the flow 


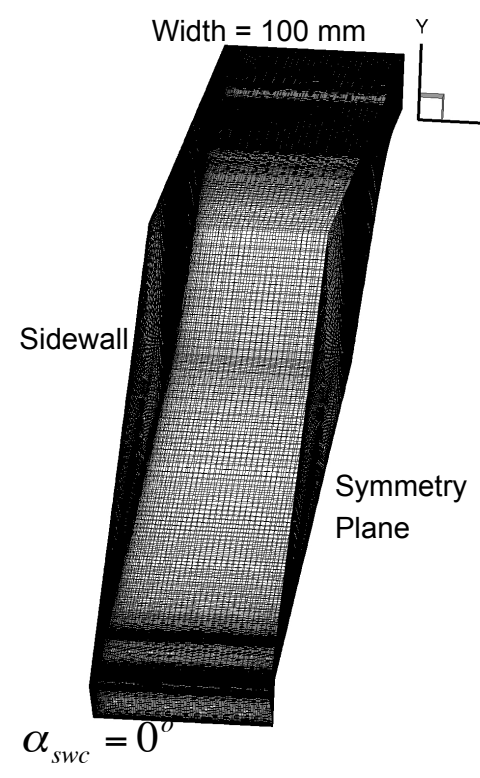

(a) No Sidewall compression

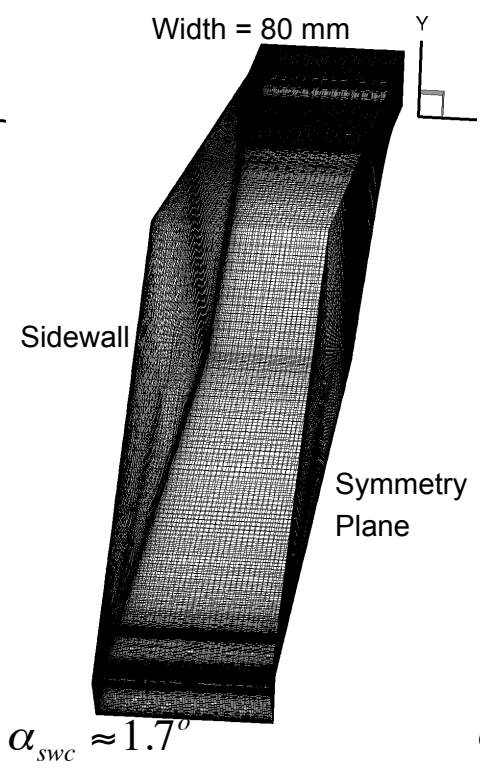

(b) $80 \mathrm{~mm} 3 \mathrm{D}$ insert

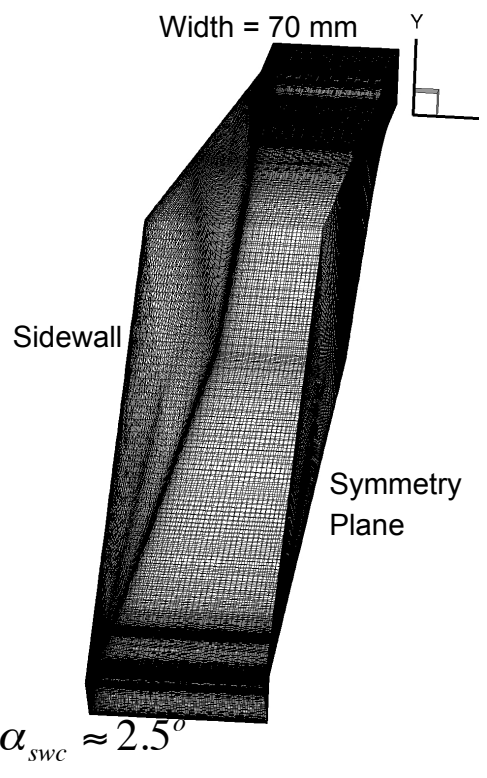

(c) $70 \mathrm{~mm} 3 \mathrm{D}$ insert

Figure 4: Grid distribution of the inlet with and without sidewall compression.

experiences supersonic expansion and relaminarization at the end of the last ramp before encountering an oblique shockwave from the cowl lip. The large adverse pressure gradient produced by this shock-boundary layer interaction causes the boundary layer to separate from the inlet wall.

It was found in the experiment ${ }^{6}$ that the flow was transitioning in the shear layer over the separation bubble between the first and second ramps. This phenomenon is taken into account in the simulation by using a transition box as described in the previous section. The validity of this approach is shown in Fig. 5 in which the wall pressure and the heat transfer distributions along the bottom wall of the symmetry plane of the inlet are compared with the measurements. For clarity, additional turbulent computation was also performed and included in this figure. Two distinct features are obvious in the computation with transition box: the flow is laminar on the first ramp which agrees much better to the experimental data and the adverse pressure gradient due to the flow turning on the second ramp induces a flow separation which causes a small pressure plateau in the last part of the first ramp. In the fully turbulent computation, the boundary layer is less susceptible to adverse pressure gradient, thus, there is no flow separation in between the ramps.

The flow features near the sidewall are rather complicated. Here, the interaction can be classified as intake-type corner flow ${ }^{17}$ in which the strong ramp shock interacts with the weak shock wave produced by the sidewall and impinges on the boundary layer that is developing on the adjacent surface. However, since the sidewall shock is very weak in comparison to the ramp shock in this case, the interaction may also be viewed as a fin-type configuration. Alvi and Settles ${ }^{18}$ proposed a model of the flow structure for this type of interaction based on flow visualization (see Fig. 6a). Along the flat plate, the fin shock is acting as a swept shock wave that turns, compresses and decelerates the near wall flow. The degree of turning is not the same throughout the whole boundary layer because of the non uniform Mach number. This causes the formation of a vortical motion that sweeps the high momentum fluid in the outer layer to the wall and remove the low momentum fluid from the wall. ${ }^{19}$ The inviscid shock bifurcates into a separation shock and a rear shock and a slip line is emanated from the triple point in order to make sure that the pressure and flow direction are the same downstream of the $\lambda$ shock structure. Furthermore, in between the slip line and the primary vortex, the flow is first accelerated through a series of expansion fan before becoming a high speed impinging jet in the near wall region. All the main features in the Alvi and Settles' model ${ }^{18}$ can be observed in the current computation as illustrated in the Mach and total pressure contour plots in Fig. 6. This plot is taken from a plane normal to the free stream direction, at a certain distance downstream of the leading edge of the first ramp. The $\lambda$ shock, the expansion region and the primary vortex are visible in the Mach contour 

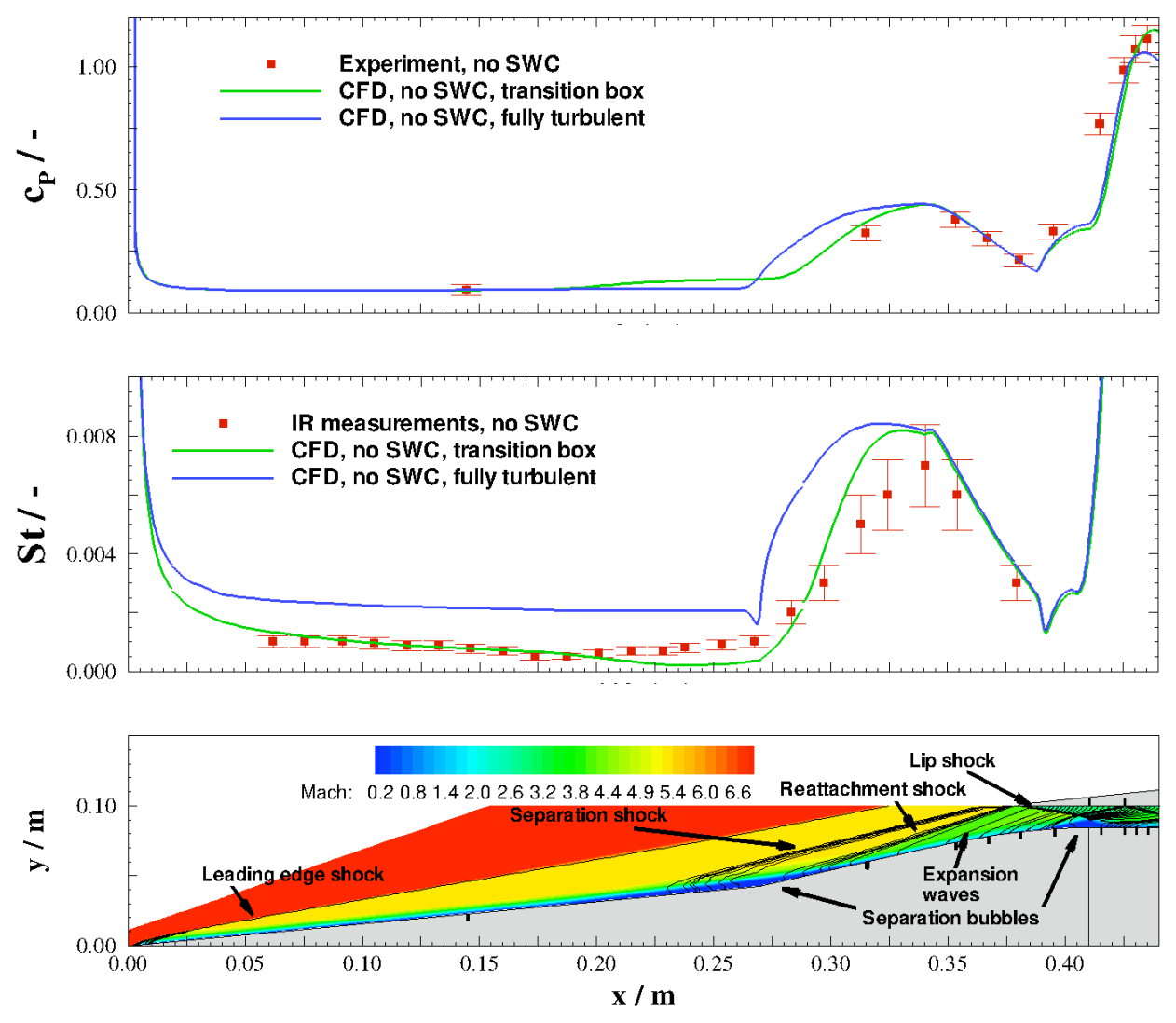

Figure 5: Pressure and heat transfer along the bottom wall, in the symmetry plane of the inlet without sidewall compression. In the bottom plot, the background is the Mach contours and the solid lines are the iso-pressure lines.

plot while the slip line and the jet impingement are more clearly shown in the total pressure plot. At the wall, the impinging jet diverges underneath the primary vortex and the corner between the ramp and the sidewall and initiates two additional secondary vortices. These secondary vortices were observed in both experimental and numerical works by Kubota and Stollery ${ }^{20}$ and Knight et al. ${ }^{21}$

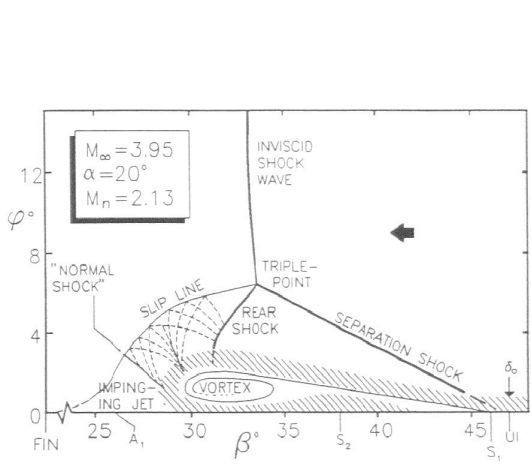

(a) Alvi \& Settles' model ${ }^{18}$

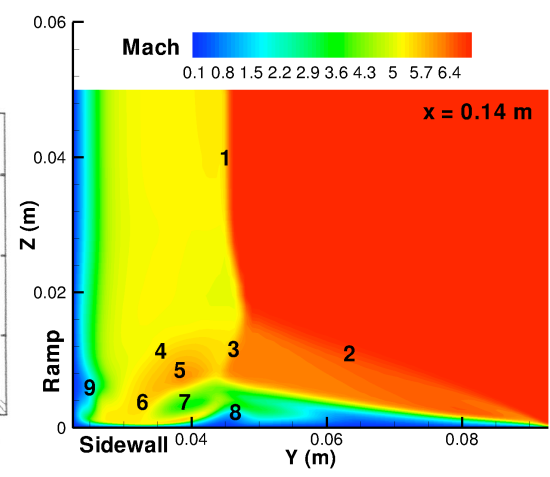

(b) CFD, Mach number

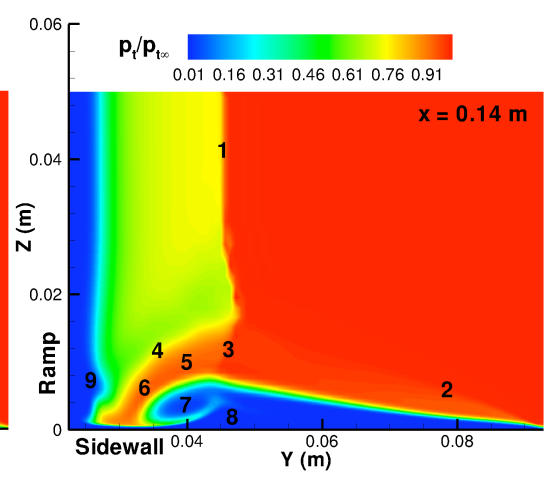

(c) CFD, total pressure

Figure 6: Comparison of Alvi and Settles' model ${ }^{18}$ and the flow structures near the sidewall in the inlet. Several flow features are shown: 1) ramp shock, 2) separation shock, 3) rear shock, 4) slip line, 5) expansion region, 6) impinging jet, 7) primary vortex, 8) and 9) secondary vortices. 
Fig. 7 shows the footprint of the flow structures on the ramps and the sidewall in terms of Stanton number. On the first ramp, near the symmetry plane, the low Stanton number region (region 1) represents the separation bubble. The reattachment region (region 2) is quite obvious on the second ramp which is a region of increasing heat transfer. This region is interrupted at the onset of the isentropic expansion surface where the flow is relaminarized and the Stanton number drops (region 3). At the corner between the ramps and the sidewall, the flow is heated in the vicinity of the corner due to the divergence of the jet impingement. Next to it, the presence of the corner (secondary) vortex leaves a trace of a low heat transfer area (region 7). The surface structure on the ramps is in qualitatively agreement with the infrared picture taken during the experiment (see Fig. 7b), except the very high heat transfer region at the corner on the second ramp. The absence of this region in the infrared picture is probably due to the selected scaling, as explained by Häberle and Gülhan. ${ }^{6}$

Along the sidewall surface, the footprints of the sidewall separation and the jet impingement are visible through a region of low Stanton number (region 4) and a region of high Stanton number (region 5), respectively. The sidewall separation denotes the beginning of the formation of the primary vortex while the jet impingement defines a region beyond which the flow is approximately parallel to the ramp. ${ }^{22}$ In addition, the trace of the secondary vortex can also be seen as a low heat transfer region (region 6) in between regions 4 and 5 . In the baseline configuration, the shock waves associated with the second ramp do not change the flow features near the sidewall dramatically. The most noticeable effect observed in the numerical results is that the impinging jet becomes significantly stronger and amplifies the heat transfer along the sidewall.

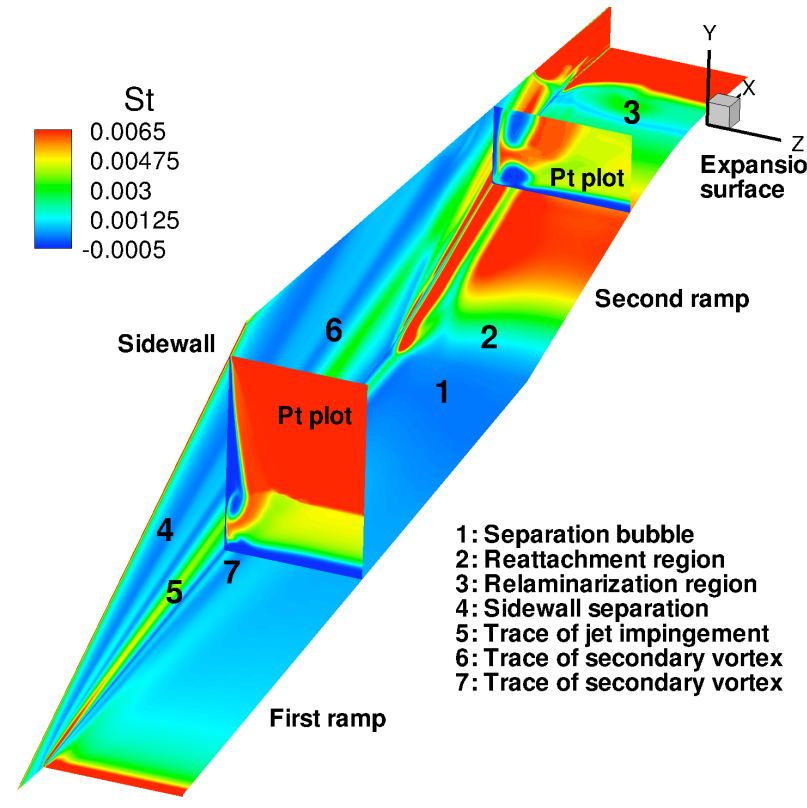

(a) CFD (half width)

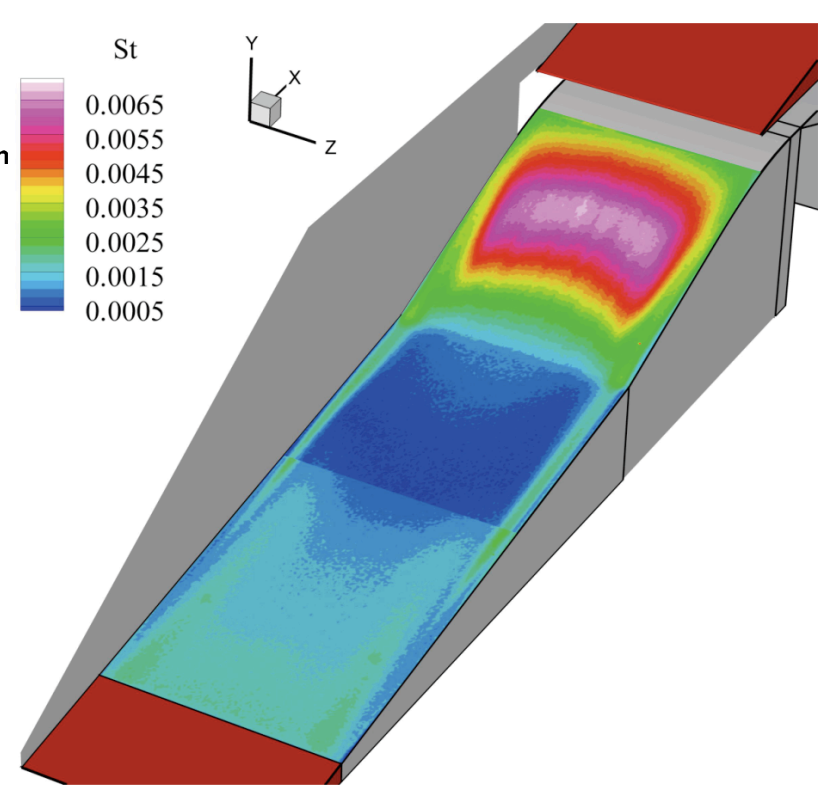

(b) Infrared picture (full width)

Figure 7: Heat transfer (Stanton number) contours on the ramp and the sidewall. "Pt plot" refers to the total pressure ratio plot as shown in Fig. 6 .

\section{Effects of Sidewall Compression}

In this section, the inlet with sidewall compression is considered. The top plot in Fig. 8 illustrates the computed wall pressure along the bottom wall in the symmetry plane. Similar to the baseline configuration, the flow is assumed to be laminar on the first ramp. Here the numerical results are in good agreement with the measurements and the assumption seems to be acceptable. As expected, increasing the sidewall compression increases the pressure in the inlet. The earlier rise of the pressure on the first ramp at larger sidewall compression indicates that the flow separation at the kink between the first ramp and the second ramp is more severe. This is not surprising since, in case of sidewall compression, the streamtube is compressed 
by both the ramps and the sidewall convergence, streamwise pressure gradient is stronger and the laminar boundary layer separates earlier on the first ramp. However, the measurements were not sufficient to conclude that the size of the separation bubble was predicted quantitatively accurately in the computations.

The flow features in the symmetry plane of the inlet with 70-mm insert are shown in the middle plot in Fig. 8. Consistent with the discussion above, the separation bubble in between the ramps is much larger in comparison to the inlet without sidewall compression (see Fig. 5). This causes the separation shock to move significantly upstream and eventually hits the leading edge shock. The interaction results in a deflected shock and a slip line downstream of the triple point. In order to assess the influence of the separation bubble, the flow features of an additional fully turbulent computation are illustrated in the bottom plot in Fig. 8. Since there is no flow separation in this case, the additional compression is achieved more gradually through a series of compression waves on the first ramp and the final deflected shock wave is weaker than that in the transition box computation.
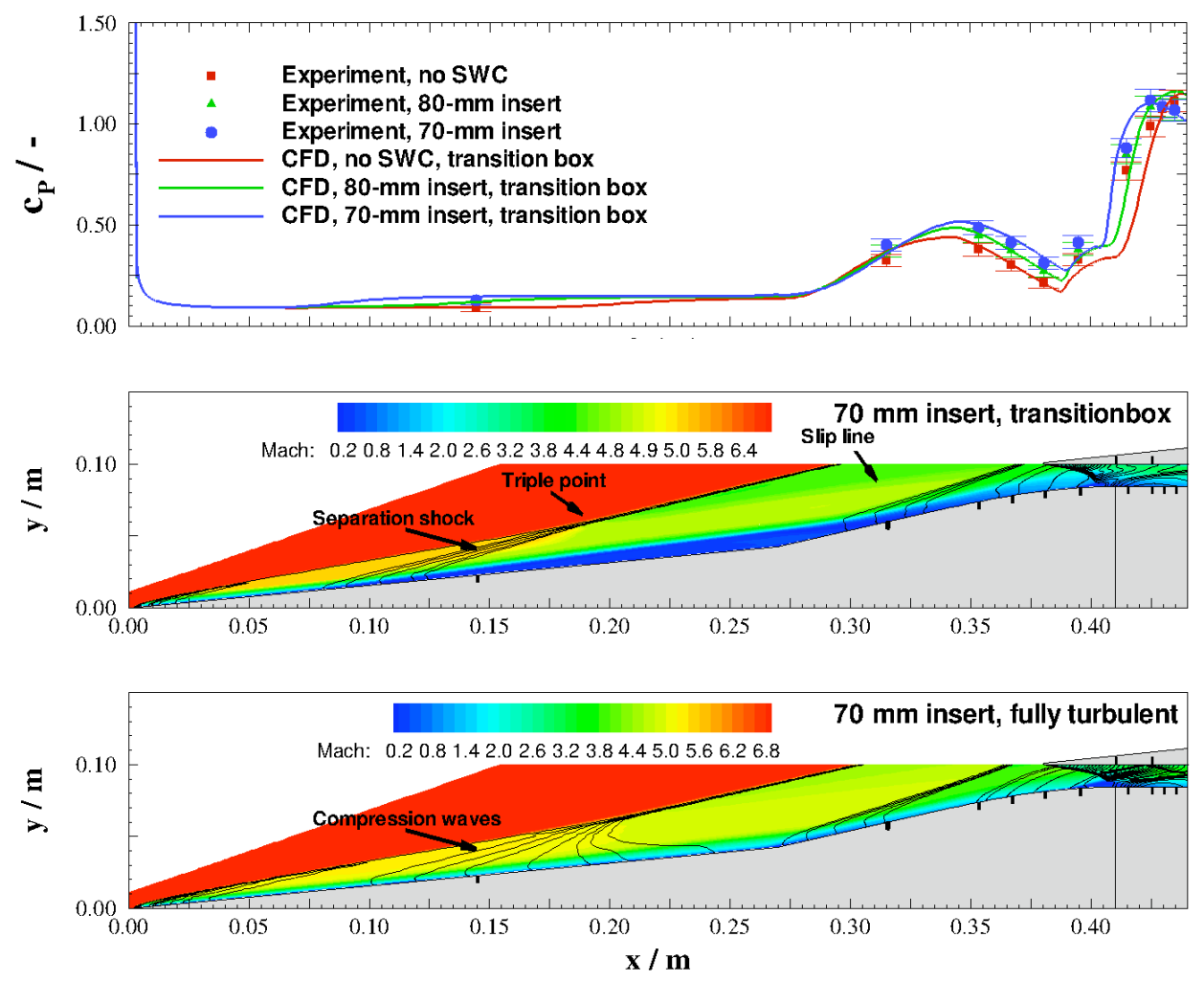

Figure 8: Top plot: pressure coefficient along the bottom wall in the symmetry plane. Middle and bottom plots: the background is the Mach contours and the solid lines are the iso-pressure lines.

It is now interesting to examine how the presence of sidewall compression modifies the flow structures and imposes more compression in the symmetry plane. Fig. 9 shows the flow field in planes normal to the free-stream direction at three different streamwise locations. For the case with sidewall compression, results from fully turbulent computations are also included to isolate the effects of the separation bubble. At the first station $(\mathrm{x}=0.07 \mathrm{~m})$, in the baseline configuration, the ramp shock is slightly curved upward in the vicinity of the triple point. This curve is due to the requirement that pressure must be equal across the slip line. Here, the ramp shock is equivalent to a "Mach stem" in 2D Mach reflection. ${ }^{23}$ With sidewall compression, the flow must be compressed more in the region close to the sidewall and this is achieved by a larger curve which extends further towards the symmetry plane. This curve is analogous to a "Mach disk" in a typical symmetric corner flow. ${ }^{17}$ The streamwise flow (normal to the Y-Z plane) below this curve is deflected by an angle larger than the ramp angle and the increase of pressure across the shock wave is larger. Furthermore, the vortex is flattened and the associated flow structures are more confined to the sidewall region. This station is upstream of the laminar separation so the flow fields in the transition box computation and fully 
turbulent computation are almost identical.

At the next station $(\mathrm{x}=0.2 \mathrm{~m})$, the effect of sidewall compression is extended to the symmetry plane. The ramp shock is being curved more significantly in comparison to the baseline configuration which allows a greater compression as explained before. Along the streamwise direction, this must be accompanied by a series of compression waves on the ramp like what seen in the bottom plot in Fig. 8. This causes the boundary layer to separate earlier if the flow is laminar on the first ramp and results in a larger separation bubble. The bubble, in return, causes a strong separation shock to deflect the flow around the separation streamline. Thus, the ramp shock is bent even more upward. This phenomenon can be seen by comparing the ramp shock in the transition box computation to that in the fully turbulent computation.

The contour at $\mathrm{x}=0.28 \mathrm{~m}$ further confirms all the observations above. Since the whole system has a tendency to deflect the flow more upward, the mass flow spillage is increased as shown in Table 2. In this table, the mass flow capture is defined as the ratio between the mass flow rate inside the interior part and the available mass flow rate from the free-stream. The pressure ratios are the ratios between the pressures at the end of the computational domain $(\mathrm{x}=440 \mathrm{~m})$ and the free-stream values. As expected, the captured mass flow is smaller and the static pressure is larger as the sidewall compression is increased. The total pressure ratio remains unchanged for the inlet with $80 \mathrm{~mm}$ insert but is lower for the inlet with $70 \mathrm{~mm}$ insert which means that the sidewall compression causes more losses in the system.

\begin{tabular}{|l|l|l|l|}
\hline & No SWC & $80 \mathrm{~mm}$ insert & $70 \mathrm{~mm}$ insert \\
\hline Mass flow capture, experiment (\%) & $73 \% \div 74 \%$ & $60 \% \div 62 \%$ & $46 \% \div 54 \%$ \\
\hline Mass flow capture, CFD (\%) & $74 \%$ & $63 \%$ & $56 \%$ \\
\hline Area-averaged static pressure ratio, CFD (-) & 25.4 & 28.3 & 30.0 \\
\hline Mass-averaged total pressure ratio, CFD (-) & 0.244 & 0.244 & 0.237 \\
\hline
\end{tabular}

Table 2: Inlet performance without and with sidewall compression. The pressure ratio is defined as the ratio of the exit pressure at $\mathrm{x}=440 \mathrm{~mm}$ and the free-stream presure.

\section{Effects of Relaminarization}

As mentioned previously, in order to assess the effects of rapid expansion and relaminarization, experiments at two different Reynolds numbers were performed in which the cowl was removed from the inlet. This is to ensure that the flow downstream of the expansion surface can be studied without any disturbances. The two bottom plots in Fig. 10 illustrate the general flow field in this case. In the absence of the cowl, the flow downstream of the second ramp gradually expands and is accelerated across the shoulder. Since the density drops more quickly than the increase of velocity, the boundary layer becomes much thicker downstream of the interaction. Both the wall pressure and the heat transfer are decreased dramatically along the expansion surface. The predicted wall pressure in the symmetry plane is in excellent agreement with the measurements whereas the peak heating rate upstream of the expansion is generally over-predicted. This is because the actual wall temperature did not remain constant during the experiment. While the assumption of constant wall temperature may seem to be valid due to short run time (approximately 20 seconds), there are certain hot spots, such as the reattachment point on the second ramp, at which the wall temperature rose significantly during the test. Since the wall temperature is fixed at $300 \mathrm{~K}$ in the computation, this explains the discrepancy at the peak heating rate. Downstream of this peak, on the expansion surface, the decrease of Stanton number is predicted reasonably accurate.

Here, it can be seen that increasing the Reynolds number has several effects on the flow. At higher Reynolds number, the boundary layer thickness is smaller on the first ramp and this causes a slightly weaker leading edge shock. The Stanton number on the first ramp is also smaller because the free-stream density, free-stream velocity and the total temperature were increased to achieve a higher Reynolds number (see Eqn. 1 for the definition of Stanton number). Since the boundary layer is thinner, the flow in the near wall region has relatively higher momentum to resist the adverse pressure gradient due to the flow turning in between the first and second ramps. Thus, the onset of the laminar separation on the first ramp is delayed and the reattachment on the second ramp is earlier, as indicated in the wall pressure and contour plots (Fig. 10). This is consistent with the flow features observed in the infrared picture by Häberle and 
Mach: 0.21 .22 .23 .24 .25 .26 .2
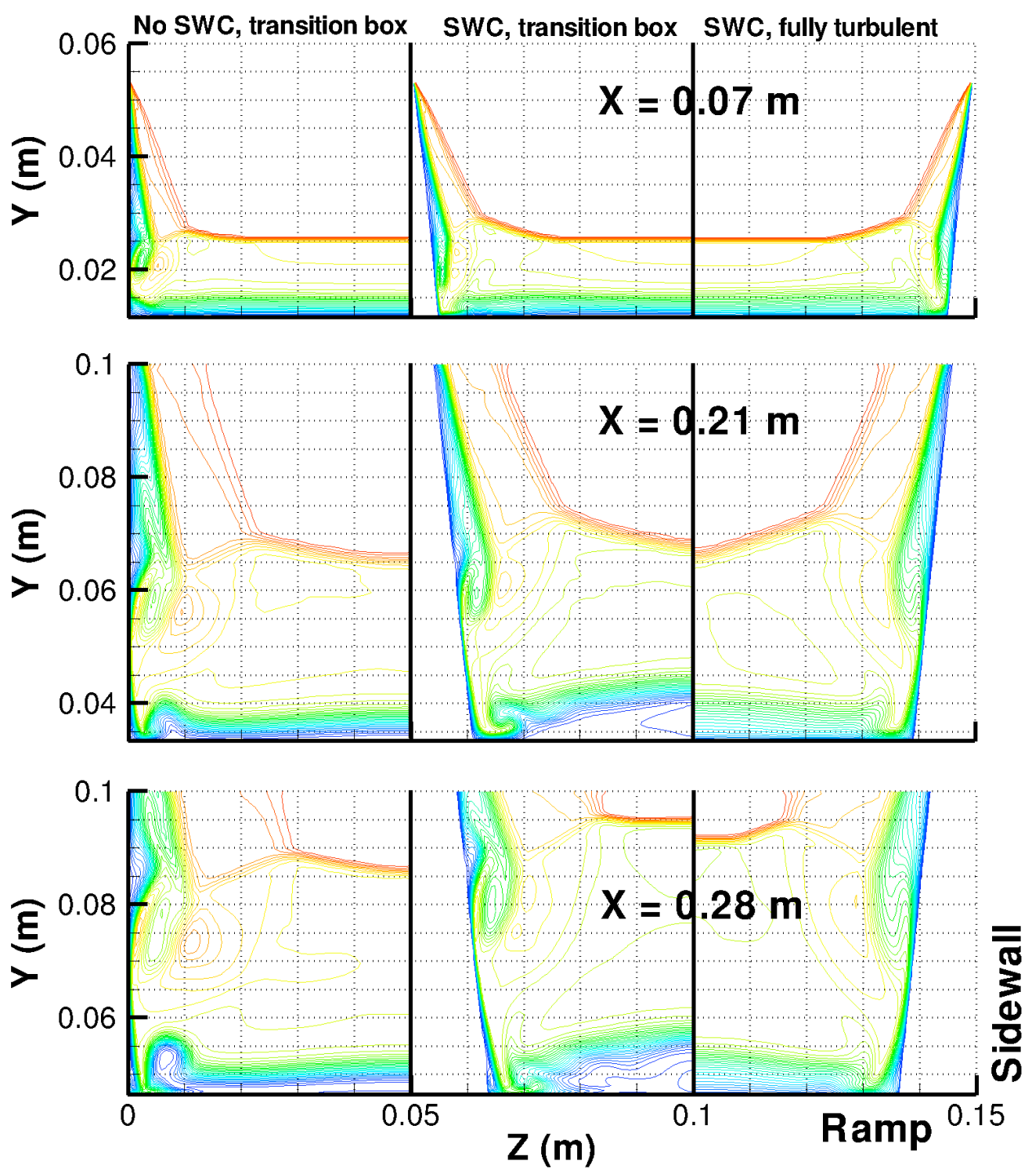

Figure 9: Mach contours in planes normal to the free-stream direction. SWC refers to the inlet with $70 \mathrm{~mm}$ insert.

Gülhan. ${ }^{6}$ Downstream of the reattachment, the Stanton number distribution forms a plateau at which the peak heating rate remains constant and its value is considerably lower than that at lower Reynolds number. At both Reynolds number, the Stanton numbers drop to a similar minimum value from the peak value indicating that across an expansion region, the reduction of heat transfer is less when the Reynolds number is larger.

The phenomenon of relaminarization will now be analyzed closer. An additional computation in which the boundary layer is assumed to be fully laminar downstream of the expansion surface $(\mathrm{x}>0.38 \mathrm{~m})$ was performed and the results are included in Fig. 10 (labelled as "lam. down."). This helps to quantify how low the Stanton number would be if the flow was fully relaminarized. The heat transfer in this ideal case is slightly smaller than that in reality which indicates that the actual flow is quasi-laminar. Fig. 11a illustrates that the turbulence kinetic energy is decreased significantly across the expansion waves and further downstream. 

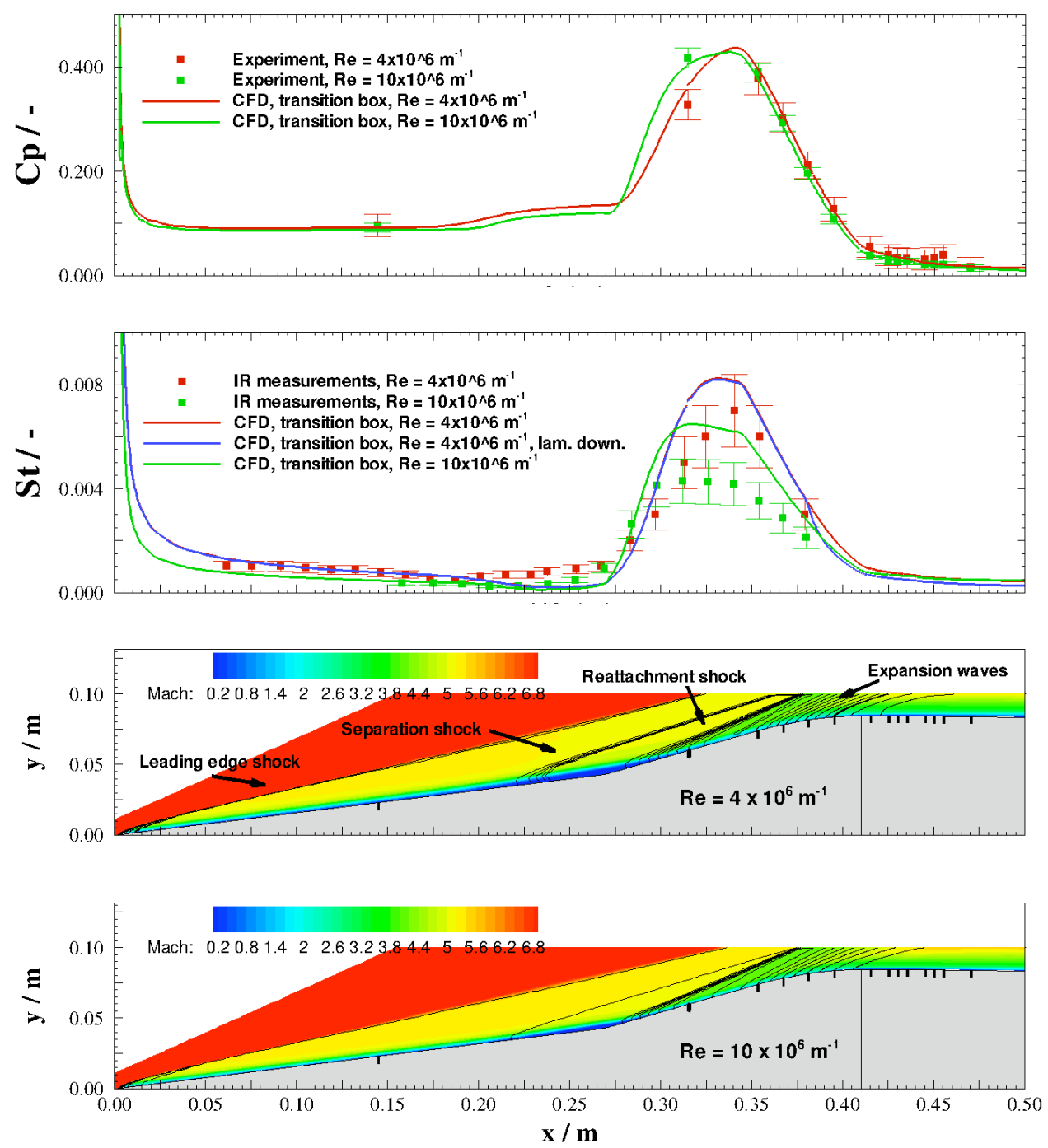

Figure 10: Pressure and heat transfer along the bottom wall, in the symmetry plane of the inlet with the cowl removed. In the two bottom plots, the background is the Mach contours and the solid lines are the iso-pressure lines.

This reduction is a combined effect of pressure gradient, streamline curvatures and bulk dilatation (change of mean density). It was shown experimentally ${ }^{3}$ and numerically ${ }^{5}$ that bulk dilatation is the most important factor and is more dominant when the expansion is stronger. These works also showed that the velocity profile downstream of the expansion region departs from the log-law in which the laminar viscous sub-layer is extended, the buffer layer overshoots the log line and the wake layer collapses. The extension of the laminar viscous sub-layer supports the proposal of the formation of a new laminar sub-layer by Narasimha and Sreenivasan. ${ }^{24}$ In order to illustrate this, the importance of laminar viscosity in relative to the turbulent viscosity is shown in Fig. 11b. Upstream of the expansion region, it can be seen that the turbulent viscosity is much larger than the laminar one, except in the vicinity of the solid wall. Due to both the suppression of turbulence and the expansion of the boundary layer, the region in which the laminar viscosity is important is enlarged significantly downstream of the inlet shoulder and this is a clear sign of the new laminar sub-layer. Since laminar boundary layer is more susceptible to flow separation, this thick laminar sub-layer would be partially responsible for the large separation bubble at the entrance of the interior part if the cowl was not removed. 


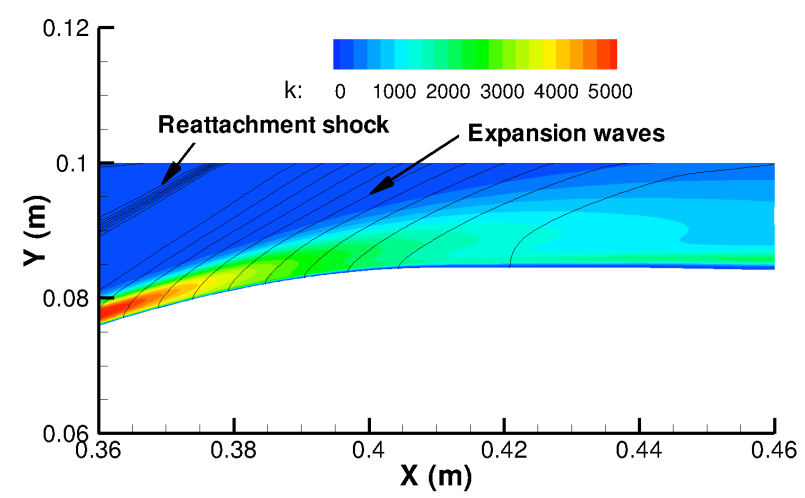

(a) Turbulence kinetic energy

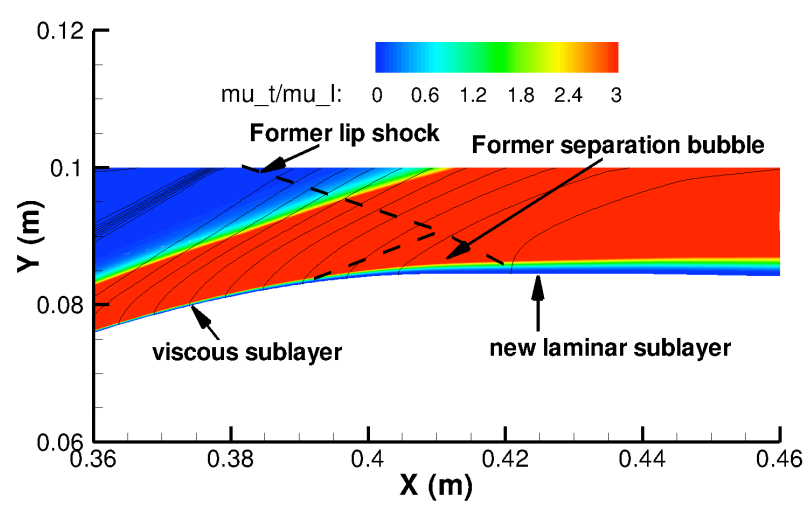

(b) Viscosity ratio

Figure 11: The effects of rapid expansion on turbulence in the scramjet inlet with cowl removed. The background is the contour plot of (a) the turbulence kinetic energy or (b) viscosity ratio. The solid lines are the iso-pressure lines and the dashed lines are the former flow features when the cowl was installed.

\section{Conclusions}

Computations were carried out to assess the effects of sidewall compression and relaminarization on the performance of a 3D scramjet inlet. Sidewall compression was achieved by introducing a smooth contraction along the outer part of the inlet. Excellent agreement with the wall pressure measurements were observed and the flow features suggest that a convergent sidewall compresses the flow by curving the ramp shock upward in the vicinity of the sidewall and extending this curve towards the symmetry plane. It was also demonstrated that this phenomenon would be enhanced if the flow separated on the ramp. The numerical results indicate that sidewall compression alter the inlet performance significantly. While the static pressure is increased due to more compression, both the mass flow capture and the total pressure ratio are reduced. This is because the shock system has a tendency to deflect the flow more upward and there are more pressure losses due to stronger shock wave and larger separation bubble.

Relaminarization was studied experimentally and numerically by testing the inlet with the cowl removed at two different Reynolds number. The computations predict the measured wall pressure accurately. The predicted heat transfer is acceptable except around the reattachment point on the second ramp where the peak heating rate is over-predicted. This is because the wall temperature was heated significantly in the experiment and this was not taken into account in the computations. Downstream of the expansion surface, both the Stanton number and the turbulence kinetic energy are reduced dramatically. The flow is found to be quasi-laminar and there is a formation of a thick viscous sub-layer. At higher Reynolds number, the overall reduction of heat transfer is smaller, indicating that the flow is less relaminarized.

Several phenomena have been learned in this work that are useful in designing a scramjet inlet. First of all, inlet designer should beware that sidewall compression causes the ramp shock to bent more upward and if the location of the cowl lip is unchanged, more mass flow spillage will occur. Secondly, due to higher pressure gradient, the boundary layer inside the inlet will be more likely to separate and results in significant reduction of performance. This may be avoided if the boundary layer is turbulent as illustrated in the previous section. Finally, since relaminarization causes the formation of a thick laminar sub-layer, the boundary layer is prone to strong separation under adverse pressure gradient due to shock wave boundary layer interaction. Thus, it is probably a good idea to impose the shock wave upstream of the expansion region where the boundary layer is still turbulent so that the probability of massive flow separation is reduced.

\section{Acknowledgments}

We would like to acknowledge Professor A. J. Smits for suggesting useful literature on swept shock boundary layer interaction. This work was supported by the German Research Foundation (DFG) within the framework of the GRK 1095 "Aero-Thermodynamic Design of a Scramjet Propulsion System for Future 
Space Transportation Systems" and the GSC 111 Aachen Institute for Advanced Study in Computational Engineering Science (AICES, RWTH Aachen University). Computing resources were provided by the RWTH Aachen University Center for Computing and Communication and the Forschungszentrum Jülich.

\section{References}

${ }^{1}$ Weigand, B., Gaisbauer, U., Reinartz, B., Kau, H.-P., and Schröder, W., "Das Graduiertenkolleg 1095/1: AeroThermodynamische Auslegung eines Scramjet-Antriebssystems füer zukünftige Raumtransportsysteme," Proceedings of Deutscher Luft- und Raumfahrtkongress 2006, DGLR, Braunschweig, Germany, November 6-9, 2006.

${ }^{2}$ Holland, S. D., "Computational Parametric Study of Sidewall-Compression Scramjet Inlet Performance at Mach 10," NASA Technical Memorandum 4411, February 1993.

${ }^{3}$ Dussauge, J. P. and Gaviglio, J., "The Rapid Expansion of a Supersonic Turbulent Flow: Role of Bulk Dilatation," Journal of Fluid Mechanics, Vol. 174, 1987, pp. 81-112.

${ }^{4}$ Smith, D. R. and Smits, A. J., "The Rapid Expansion of a Turbulent Boundary Layer in a Supersonic Flow," Theor. Comput. Fluid Dyn., Vol. 2, 1991, pp. 319-328.

${ }^{5}$ Nguyen, T., Behr, M., and Reinartz, B., "Numerical Investigation of Compressible Turbulent Boundary Layer Over Expansion Corner," AIAA Paper 2009-7371, 2009.

${ }^{6}$ Häberle, J. and Gülhan, A., "Investigation of Two-Dimensional Scramjet Inlet Flowfield at Mach 7," Journal of Propulsion and Power, Vol. 24, No. 3, 2008, pp. 446-459.

${ }^{7}$ Hohn, O. and Gülhan, A., "Experimental Investigation on the Influence of Sidewall Compression of the Flowfield of a Scramjet Inlet at Mach 7," to be published, 2010.

${ }^{8}$ Hohn, O. and Gülhan, A., "Experimental Investigation on the Influence of Yaw Angle on the Inlet Performance at Mach 7," AIAA Paper 2010-938, 2010.

${ }^{9}$ Wada, Y. and Liou, M. S., "An Accurate and Robust Flux Splitting Scheme for Shock and Contact Discontinuities," SIAM Journal on Scientific Computing, Vol. 18, 1997, pp. 633-648.

${ }^{10}$ Bramkamp, F. D., Lamby, P., and Müller, S., "An adaptive multiscale finite volume solver for unsteady and steady flow computations," Journal of Computational Physics, Vol. 197, 2004, pp. 460-490.

${ }^{11}$ Krause, M. and Ballmann, J., "Numerical Simulations and Design of a Scramjet Intake Using Two Different RANS solver," AIAA Paper 2007-5423, 2007.

${ }_{12}$ Menter, F. R., "Two-Equation Eddy-Viscosity Turbulence Models for Engineering Applications," AIAA Journal, Vol. 32, No. 8, Aug. 1994, pp. 1598-1605.

${ }^{13}$ Krause, M. and Behr, M., "Modeling of Transition Effects in Hypersonic Intake Flows Using a Correlation-Based Intermittency Model," 15th AIAA International Space Planes and Hypersonic Systems and Technologies Conference, Dayton (OH), USA, 28 April - 1 May 2008.

${ }^{14}$ Krause, M. and Ballmann, J., "Enhanced Design of a Scramjet Intake Using Two Different RANS Solvers," 26th International Symposium on Shock Waves, Goettingen, Germany, 15-20 July 2007, 2007, pp. 589-594.

${ }^{15}$ Brodersen, O., Ronzheimer, A., Ziegler, R., Kunert, T., Wild, J., and Hepperle, M., "Aerodynamic Applications using MegaCads," Proc. of 6th International Conference on Numerical Grid Generation in Computaional Field Simualtions, Ed. M. Cross, J. Haeuser, B. K. Soni, J. F. Thompson, ISGG 1998, 1998, pp. 793-802.

${ }^{16}$ Häberle, J. and Gülhan, A., "Experimental Investigation of a Two-Dimensional and a Three-Dimensional Scramjet Inlet at Mach 7," Journal of Propulsion and Power, Vol. 24, No. 5, Sep.-Oct. 2008, pp. 1023-1034.

${ }^{17}$ D'Ambrosio, D. and Marsilio, R., "Shock-Induced Separated Structures in Symmetric Corner Flows," NASA Contractor Report 198239, December 1995.

${ }^{18}$ Alvi, F. S. and Settles, G. S., "Physical Model of the Swept Shock Wave/Boundary-Layer Interaction Flowfield," AIAA Journal, Vol. 30, 1992, pp. 2252-2258.

${ }^{19}$ Smits, A. J. and Dussauge, J.-P., Turbulent Shear Layers in Supersonic Flow, chap. 10, Springer Science + Business Media, Inc, 2006.

${ }^{20}$ Kubota, H. and Stollery, J., "An Experimental Study of the Interaction Between a Glancing Shock Wave and a Turbulent Boundary Layer," Journal of Fluid Mechanics, Vol. 116, 1982, pp. 431-458.

${ }^{21}$ Knight, D. D., Badekas, D., Horstman, C. C., and Settles, G. S., "Quasiconical Flowfield Structure of a Three-Dimensional Single Fin Interaction," AIAA Journal, Vol. 30, 1992, pp. 2809-2816.

${ }^{22}$ Knight, D. D., Horstman, C. C., Shapey, B., and Bogdonoff, S., "Structure of Supersonic Turbulent Flow Past a Sharp Fin," AIAA Journal, Vol. 25, 1987, pp. 1331-1337.

${ }^{23} \mathrm{Lu}$, F. K., "Quasiconical Free Interaction between a Swept Shock and a Turbulent Boundary Layer," AIAA Journal, Vol. 31, 1993, pp. 686-692.

${ }^{24}$ Narasimha, R. and Sreenivasan, K. R., "Relaminarization in Fluid Flows," Advances in Applied Mechanics, Vol. 19, 1979, pp. 221-309. 